\title{
Improving the diagnosis of common parotid tumors via the combination of CT image biomarkers and clinical parameters
}

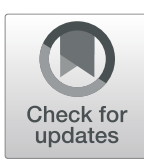

Dan Zhang ${ }^{1,2}$, Xiaojiao Li ${ }^{1,2}$, Liang Lv', Jiayi Yu ${ }^{1,2}$, Chao Yang ${ }^{1,2}$, Hua Xiong ${ }^{1,2}$, Ruikun Liao ${ }^{1,2}$, Bi Zhou ${ }^{1,2}$, Xianlong Huang ${ }^{1}$, Xiaoshuang Liu ${ }^{3}$ and Zhuoyue Tang ${ }^{1,2^{*}}$

\begin{abstract}
Background: Our study aims to develop and validate diagnostic models of the common parotid tumors based on whole-volume $C T$ textural image biomarkers (IBMs) in combination with clinical parameters at a single institution.

Methods: The study cohort was composed of 51 pleomorphic adenoma (PA) patients and 42 Warthin tumor (WT) patients. Clinical parameters and conventional image features were scored retrospectively and textural IBMs were extracted from CT images of arterial phase. Independent-samples t test or Chi-square test was used for evaluating the significance of the difference among clinical parameters, conventional CT image features, and textural IBMs. The diagnostic performance of univariate model and multivariate model was evaluated via receiver operating characteristic (ROC) curve and area under ROC curve (AUC).

Results: Significant differences were found in clinical parameters (age, gender, disease duration, smoking), conventional image features (site, maximum diameter, time-density curve, peripheral vessels sign) and textural IBMs (mean, uniformity, energy, entropy) between PA group and WT group $(P<0.05)$. ROC analysis showed that clinical parameter (age) and quantitative textural IBMs (mean, energy, entropy) were able to categorize the patients into PA group and WT group, with the AUC of 0.784, 0.902, 0.910, 0.805, respectively. When IBMs were added in clinical model, the multivariate models including age-mean and age-energy performed significantly better than the univariate models with the improved AUC of 0.940, 0.944, respectively $(P<0.001)$.

Conclusions: Both clinical parameter and $C T$ textural IBMs can be used for the preoperative, noninvasive diagnosis of parotid PA and WT. The diagnostic performance of textural IBM model was obviously better than that of clinical model and conventional image model in this study. While the multivariate model consisted of clinical parameter and textural IBM had the optimal diagnostic performance, which would contribute to the better selection of individualized surgery program.
\end{abstract}

Keywords: Parotid tumors, Image biomarkers, Clinical parameters

\footnotetext{
*Correspondence: zhuoyue_tang@ucas.ac.cn

${ }^{1}$ Department of Radiology, Chongqing General Hospital, University of

Chinese Academy of Sciences, No.104 Pipashan Main St, Yuzhong District, Chongqing 400014, China

${ }^{2}$ Molecular and Functional Imaging Laboratory, Chongqing General Hospital, University of Chinese Academy of Sciences, Chongqing 400014, China

Full list of author information is available at the end of the article
} 


\section{Background}

Parotid gland neoplasms are the most common type of salivary gland neoplasms, with $75-80 \%$ being benign [1]. Pleomorphic adenoma (PA) and Warthin tumor (WT) are the two most common types of the parotid gland tumors, and the incidence of WT has been gradually increasing nowadays [1, 2]. Although both of them are benign tumors, the biological characteristics and surgery programme are completely different. PA may develop into malignant tumor as a result of a delayed surgery, and postoperative recurrence of PA is very common. Yet, WT grows slowly, rarely recurs and malignant transformation seldom occurs [3, 4]. At present, partial superficial parotidectomy (PSP) is the most common surgical procedure for PA and extracapsular dissection (ECD) for WT, which is in line with the current trend of minimising surgical dissection. Therefore, the risk of short-term and long-term complications might be decreased [5]. ECD is a safe and time-efficient surgical approach, offering earlier recovery and better preservation of salivary function compared to PSP. Meanwhile, ECD should be considered as a surgical approach for parotid tumors, especially those in the parotid tail, such as WT [6]. Yet, ECD is not quite suitable to be applied to PA, for it will increase the postoperative recurrence rate compared to PSP.

Preoperative knowledge of the pathological type of tumors would be of great importance in consideration of optimizing the individualized operative program and help to inform preoperative patient counselling. In routine clinical practice, common factors such as age, performance status, tumor size, site, and the like, are used to guide treatment decision-making. Nevertheless, patients with similar factors above may have different outcome. Neither these clinical factors nor conventional image features are sufficient for identifying patients that will benefit most from specific surgical strategies. Thus, more detailed information that reflect the characteristics of the whole tumor are needed to improve the diagnostic accuracy.

Some recent reports have indicated that IBMs of tumors are prominently related to the pathology and prognosis $[7,8]$. IBMs can be extracted from various medical images and provide quantitative information with regard to shape, intensity and texture features of the region of interest (ROI) [9]. There is plenty of quantitative information on medical images, far beyond our current understanding. As a result, there is an increasing interest in the assessment of tumors on medical images by advanced software in order to derive additional, clinically relevant information, namely texture analysis. Texture analysis has been used to predict several kinds of clinical issues, such as tumor heterogeneity, patient prognosis, and response to therapy [10-12]. In this study, texture features were extracted from CT images and incorporated into the diagnostic model for histological classification of the two most common benign parotid tumors, WT and PA.

Many IBMs of tumors are significantly related to the outcome, but it is still unclear to what extent the addition of IBMs improves the diagnostic performance of models consisted of clinical parameters and conventional image features. Moreover, there is a relative paucity of literature as for the combined model. The aim of this study was to test whether the diagnostic performance of prediction models could be improved by the addition of IBMs compared to models based on solely clinical parameters or conventional images for WT and PA.

\section{Methods}

\section{Patient selection}

This retrospective study was approved by the local ethics review board. The informed consent was waived for this single-institution study due to its retrospective nature. All data of patients were used confidentially and anonymously. The research involved no more than minimal risk to the patients. Meanwhile, the waiver did not adversely affect the rights and welfare of the patients. Clinical and image data of all patients were obtained through medical record system and follow-up. Between January 2016 and May 2017, patients with PA or WT who underwent surgery were eligible and identified from the institution's database.

The inclusion criteria were as follows: (1) confirmed PA or WT with postoperative pathological diagnosis; (2) contrast-enhanced CT images of neck containing parotid gland obtained within 2 weeks prior to surgery; and (3) maximum diameter of lesions $\geq 1.0 \mathrm{~cm}$. The exclusion criteria were as follows: (1) CT images with obvious artifacts, such as false teeth artifacts, motion artifacts, etc.; and (2) lesions with scarcely solid components which are difficult for texture analysis. As a result, a total of 122 patients were identified, and 29 patients were excluded (Fig. 1). The final study population comprised 93 patients.

\section{Image acquisition}

CT scans were performed using 64/128-multidetector scanners (LightSpeed VCT; GE Healthcare, Waukesha, WI, USA) and the parameters were as follows: tube current, $150 \mathrm{~mA}$; tube voltage, $120 \mathrm{kVp}$; section thickness, $5 \mathrm{~mm}$; section interval, $5 \mathrm{~mm}$.

The scanning ranges from the base of the skull to the entrance to the thorax. The enhanced images were obtained after intravenous injection of $80-100 \mathrm{~mL}$ of nonionic contrast material $(320 \mathrm{mg} / \mathrm{mL}$; Iopamidol, Shanghai Bracco Sine Pharmaceutical Co., Ltd., Shanghai, China) at an injection rate of $3.0 \mathrm{~mL} / \mathrm{s}$, followed by a $50 \mathrm{~mL}$ saline 


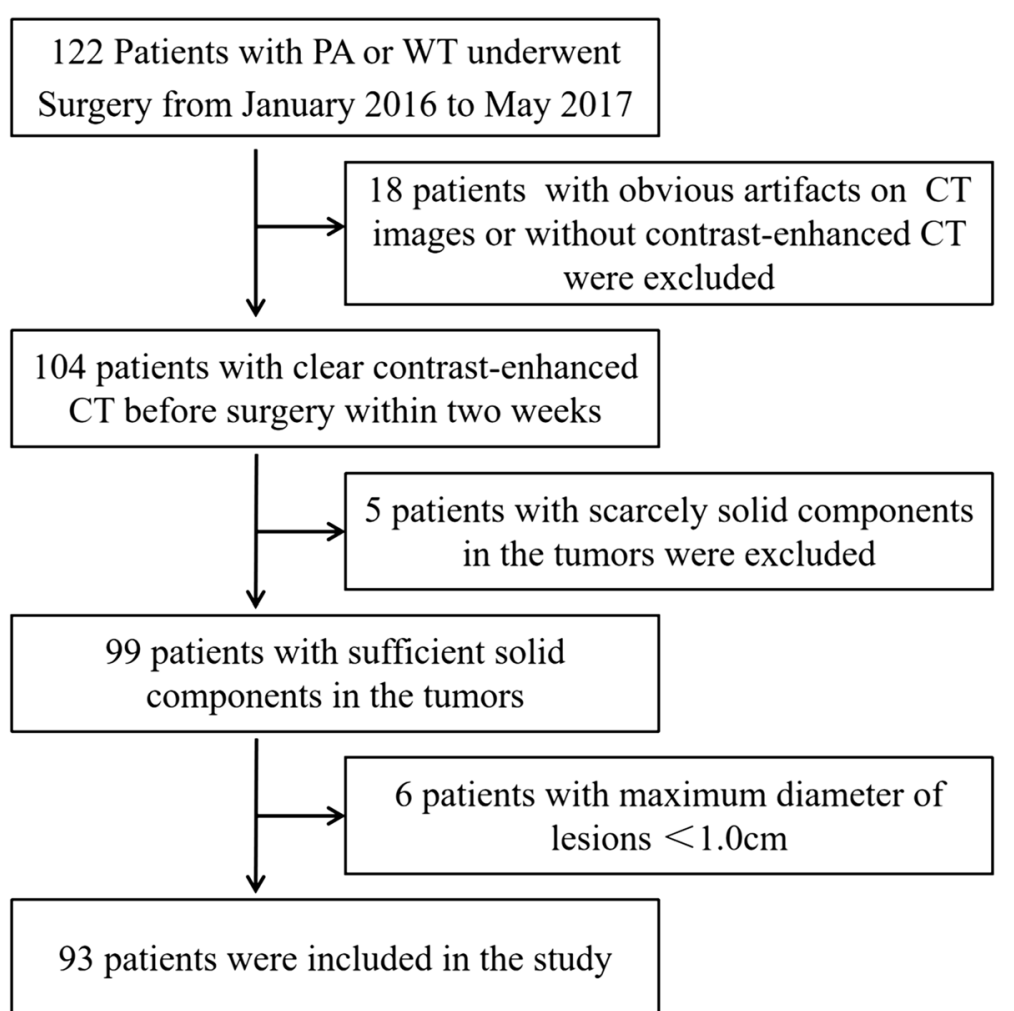

Fig. 1 Schematic shows recruitment pathway of patients for this study

chaser. The contrast-enhanced CT images were obtained at 35 and $120 \mathrm{~s}$ after contrast material injection in arterial phase and balanced phase, respectively.

\section{Clinical parameters}

All clinical parameters including age, gender, disease duration, and smoking status were collected from medical record system.

\section{Conventional CT image features}

All conventional CT image features were derived from the original CT image data, including tumor site (in the parotid tail or not), maximum diameter, time-density curve (washout type or not), and peripheral vessels sign, which defined as increased tortuous vascular shadows clinging to the edge of the lesion.

\section{CT textural image biomarkers}

CT images of arterial phase of all patients were stored in Digital Imaging and Communications in Medicine (DICOM) format and uploaded to ITK-SNAP software for three-dimensional manual segmentation of the region of interest (ROI). An ROI was manually drawn to cover the tumor as large as possible, keeping a distance of $1 \mathrm{~mm}$ from the boundary and carefully avoiding the retromandibular vein and too much parotid parenchyma into the lesion, which may lead to a misunderstanding of the internal structure of the tumor and affect the accuracy of texture analysis. The ROI of each case was manually drawn by a head and neck radiologist who did not have any knowledge about the clinical information of patients, and then the segmentation was checked by a senior radiologist. Areas of tumor heterogeneity, including cystic change or necrosis, were not excluded, for the information captured with texture analysis could potentially contribute to tumor discrimination and classification. An in-house software, Matlab2017b (Mathworks, Natick, MA, USA), was used to extract the texture parameters automatically. Six frequently-used texture parameters obtained from the gray-level histogram were included in the study, namely uniformity (a measure of the sum of the squares of each intensity value), mean (the average gray level intensity within the ROI), energy (a measure of the magnitude of voxel values in an image), entropy (the distribution of gray levels within the VOI), skewness (the histogram asymmetry degree around the mean), and kurtosis (a measurement of the histogram sharpness). An overview of the textural IBM extraction process and analysis is shown in Fig. 2.

\section{Data analysis}

The data were analyzed by SPSS 22.0 software (IBM, Chicago). Kolmogorov-Smirnov test was used for intra- 


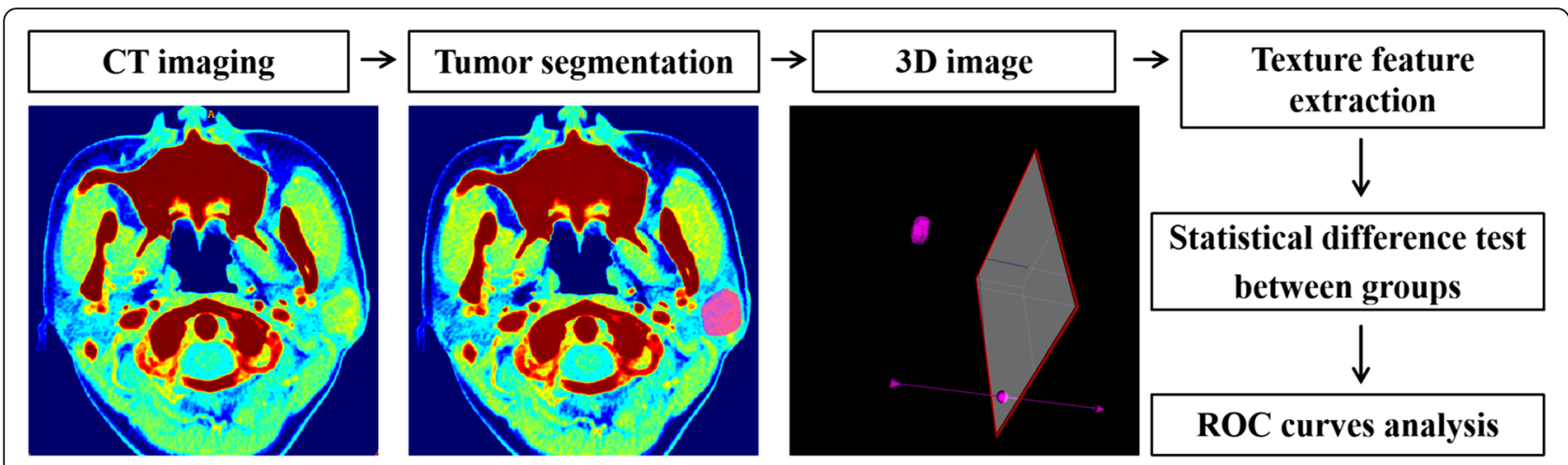

Fig. 2 Flowchart illustrating the texture analysis in this study

group normality test, and Levene test was used for intragroup variance homogeneity test. Parameters with normal distribution and homogeneity of variances were expressed as mean \pm standard deviation, and independent-samples $t$ test was adopted for data analysis. Parameters that did not satisfy normal distribution and uneven variance were expressed by median and interquartile spacing. The twoindependent-samples Mann-Whitney U Test was used for data analysis. Qualitative data were presented as ratios, which were analyzed by chi-square test. As to clinical parameter (gender) - textural IBM models, a two-component diagnostic model was fitted with binary Logistic regression analysis. The diagnostic performance of each index was tested via receiver operating characteristic (ROC) analysis. Cutoff values were established by calculating the maximal Youden index (Youden index $=$ sensitivity + specifcity- 1 ). A $P$ value was considered significant if it was less than 0.05 .

\section{Step 1 clinical model}

Potential clinical parameters that were considered for their diagnostic ability in the PA and WT datasets included age (>median vs. $\leq$ median), gender (female vs. male), disease duration (>median vs. $\leq$ median), and smoking status (yes vs. no).

\section{Step 2 conventional image model}

Potential conventional CT image features that were considered for their diagnostic ability in the PA and WT datasets included tumor site (in the parotid tail, which can be defined as inferior $2 \mathrm{~cm}$ of the superficial lobe of the gland, vs. not), maximum diameter (>median vs. $\leq$ median), time-density curve (TDC) (washout type vs. not), peripheral vessels sign, which is defined as increased tortuous vascular shadow clinging to the edge of lesion in the arterial phase (yes vs. no).

\section{Step 3 textural IBM model}

Six texture features namely mean, uniformity, energy, entropy, skewness and kurtosis were calculated and selected. The potential textural IBMs were analyzed for their diagnostic power, and the median values (>median vs. $\leq$ median) in the WT and PA datasets were regarded as the threshold value in the univariate analysis.

\section{Step 4 combined models}

According to the ROC analysis and AUC, the potential clinical parameters, conventional image features, and textural IBMs were included in the multivariate analysis to create combined models, and the optimal one was selected out.

\section{Results}

\section{Step 1 clinical model}

Univariate analysis showed the age in the WT group was significantly older than that in the PA group $(P<0.001)$. The disease duration in the PA group was significantly longer than that in the WT group $(P=0.01)$. Meanwhile, significant differences were found in gender and smoking status between the two groups $(P<0.001)$. All the selected clinical parameters showed significant differences between two groups, which was shown in Table 1.

Table 1 Baseline patient characteristics in PA goup and WT group

\begin{tabular}{llll}
\hline Characteristics & $\begin{array}{l}\text { Pleomorphic } \\
\text { adenoma } \\
(N=51)\end{array}$ & $\begin{array}{l}\text { Warthin } \\
\text { tumor } \\
(N=42)\end{array}$ & $P$ value \\
\hline $\begin{array}{c}\text { Age } \\
\text { Gender }^{\mathrm{b}} \text { (year) }\end{array}$ & $43.88 \pm 17.188$ & $59.93 \pm 6.194$ & $<0.01$ \\
$\quad \begin{array}{l}\text { Male } \\
\text { Female }\end{array}$ & $15(28.4 \%)$ & $41(97.6 \%)$ & $<0.01$ \\
Smoking status $^{\mathrm{b}}$ & $36(70.6 \%)$ & $1(2.4 \%)$ & \\
$(+)$ & $12(23.5 \%)$ & $37(88.1 \%)$ & $<0.01$ \\
$\quad(-)$ & $39(76.5 \%)$ & $5(11.9 \%)$ & \\
Disease duration $^{\mathrm{a}}$ (month) & $27.91 \pm 36.868$ & $12.54 \pm 17.105$ & 0.023 \\
\hline
\end{tabular}

N Number. (+) smoked, while (-) never smoked. ${ }^{\mathrm{a}}$ Data: Mean \pm SD. ${ }^{\mathrm{b}}$ Data: No. (percentage)

Abbreviations: PA Pleomorphic adenoma, WT Warthin tumor 


\section{Step 2 conventional image model}

The maximum diameter of lesion in the WT group was significantly larger than that in the PA group (2.41 \pm 0.534 vs. $2.02 \pm 0.588, P=0.003)$. The WT group is more inclined to be in the parotid tail compared with the PA group $(78.6 \%$ vs. $19.6 \%, P<0.001)$. As to the peripheral vessels sign and washout type of TDC, they were more frequently in the WT group compared with the PA group $(64.3 \%$ vs. 3.9 and $88.1 \%$ vs. $3.9 \%$, respectively, $P<0.001$ ) (Table 2).

\section{Step 3 textural IBM model}

The mean, energy, and entropy of WT group were significantly higher than those of PA group (all $P<0.001$ ), while the uniformity of WT group was significantly lower than that of PA group $(P<0.001)$. No statistically significant differences of skewness and kurtosis were found in both groups $(P=0.05$ and $P=0.151$, respectively) (Table 3).

\section{Step 4 diagnostic performance of univariate and multivariate models}

Univariate analysis showed significant differences in age, gender, disease duration, smoking status, site (in the parotid tail), peripheral vessels sign, TDC (washout type), maximum diameter, mean, energy, entropy and uniformity between the WT and PA group (all $P<0.05$ ). ROC analysis showed that clinical parameter (age) and textural parameters (mean, energy, entropy) performed well in differentiating the WT group from the PA group, and yielded the AUC of 0.784, 0.902, 0.910, 0.805, respectively (Figs. 3, 4, Table 4). The multivariate models were consisted of the clinical parameter and textural parameters, including age-mean, age-energy, age-entropy, with

Table 2 Conventional CT image features in PA goup and WT group

\begin{tabular}{llll}
\hline Parameter & $\begin{array}{l}\text { Pleomorphic } \\
\text { adenoma } \\
(N=51)\end{array}$ & $\begin{array}{l}\text { Warthin } \\
\text { tumor } \\
(N=42)\end{array}$ & P Value \\
\hline $\begin{array}{l}\text { Maximum diameter }{ }^{\mathrm{a}}(\mathrm{cm}) \\
\text { Site }^{\mathrm{b}} \text { (in the tail) } \\
\text { Yes }\end{array}$ & $\begin{array}{l}2.02 \pm 0.588 \\
\text { No }\end{array}$ & $2.41 \pm 0.534$ & 0.003 \\
TDC $^{\mathrm{b}}$ (washout type) & $41(80.4 \%)$ & $9(21.4 \%)$ & \\
Yes & $2(3.9 \%)$ & $37(88.1 \%)$ & $<0.01$ \\
No & $49(96.1 \%)$ & $5(11.9 \%)$ & \\
Peripheral vessels sign & & & \\
Yes & $2(3.9 \%)$ & $27(64.3 \%)$ & $<0.01$ \\
No & $49(96.1 \%)$ & $15(35.7 \%)$ & \\
\hline
\end{tabular}

$N$ Number. ${ }^{\mathrm{a}}$ Data: Mean \pm SD. ${ }^{\mathrm{b}}$ Data: No. (percentage) Abbreviations: PA Pleomorphic adenoma, WT Warthin tumor, TDC Time-density curve
Table 3 Six textural IBMs based on the CT arterial phase images in PA group and WT group

\begin{tabular}{llll}
\hline Parameter & $\begin{array}{l}\text { Pleomorphic adenoma } \\
(N=51)\end{array}$ & $\begin{array}{l}\text { Warthin tumor } \\
(N=42)\end{array}$ & $P$ Value \\
\hline Uniformity $^{\mathrm{a}}$ & $0.429 \pm 0.116$ & $0.318 \pm 0.077$ & $<0.01$ \\
Entropy $^{\mathrm{a}}$ & $1.584 \pm 0.367$ & $1.983 \pm 0.303$ & $<0.01$ \\
Mean $^{\mathrm{a}}$ & $57.664 \pm 18.121$ & $91.225 \pm 19.523$ & $<0.01$ \\
Skewness $^{\mathrm{a}}$ & $-0.455 \pm 0.568$ & $-0.678 \pm 0.613$ & 0.072 \\
Energy $^{\mathrm{a}}$ & $1.688 \mathrm{E} 7 \pm 1.708 \mathrm{E} 7$ & $7.313 \mathrm{E} 7 \pm 5.002 \mathrm{E} 7$ & $<0.01$ \\
Kurtosis $^{\mathrm{a}}$ & $3.958 \pm 1.444$ & $4.387 \pm 1.402$ & 0.152
\end{tabular}

Abbreviations: IBMs Image biomarkers, CT Computed tomography, PA Pleomorphic adenoma

${ }^{a}$ Data: Mean \pm SD

the AUC of $0.940,0.944,0.841$, respectively. The optimal multivariate models included age-mean, age-energy, and yielded the AUC, sensitivity, and specificity of 0.940 , $0.882,0.905$ and $0.944,0.922,0.857$, respectively $(P<$ 0.001) (Fig. 5, Table 5).

\section{Discussion}

This study showed a detailed analysis on the different diagnostic models for PA and WT patients. We not only calculated AUC, sensitivity and specificity of univariate and multivariate model, but also analyzed the differences between the AUC values. Our results indicated that textural IBMs might be helpful to diagnose patients with parotid PA and WT. Especially, the multivariate models including clinical parameter and textural IBMs showed better diagnostic performance in this study.

Nowadays, surgeons are in great efforts to reduce the incidence of post-parotidectomy complications under the advocacy of precision medicine. PSP and ECD are the two most commonly used surgery programmes for patients with parotid tumors at present [5, 13]. ECD is often used to excise tumors located in parotid tail. It has been considerd as a minimally invasive surgery and results in fewer complications, higher efficiency, and better preservation of salivary function $[6,14,15]$. Parotid tail is defined as inferior $2 \mathrm{~cm}$ of the superficial lobe and lies anterolateral to sternocleidomastoid muscle [16]. The surgical plan of neoplasms located in the parotid tail may be quite different from other parotid areas, especially for WT [6]. In our study, most of WT were located in the parotid tail, while few of PA were discovered in the tail $(P<0.001)$. Consequently, ECD is not quite suitable to be applied to PA. Furthermore, it has been proved a significantly higher rate of recurrent PA with ECD compared to PSP [17].

Parotid PA and WT always present as painless, slowly growing mass without characteristic performance in laboratory and traditional imaging examinations. It has been demonstrated that smoking and elderly male were characteristics of WT, with the ratio of male patients to 

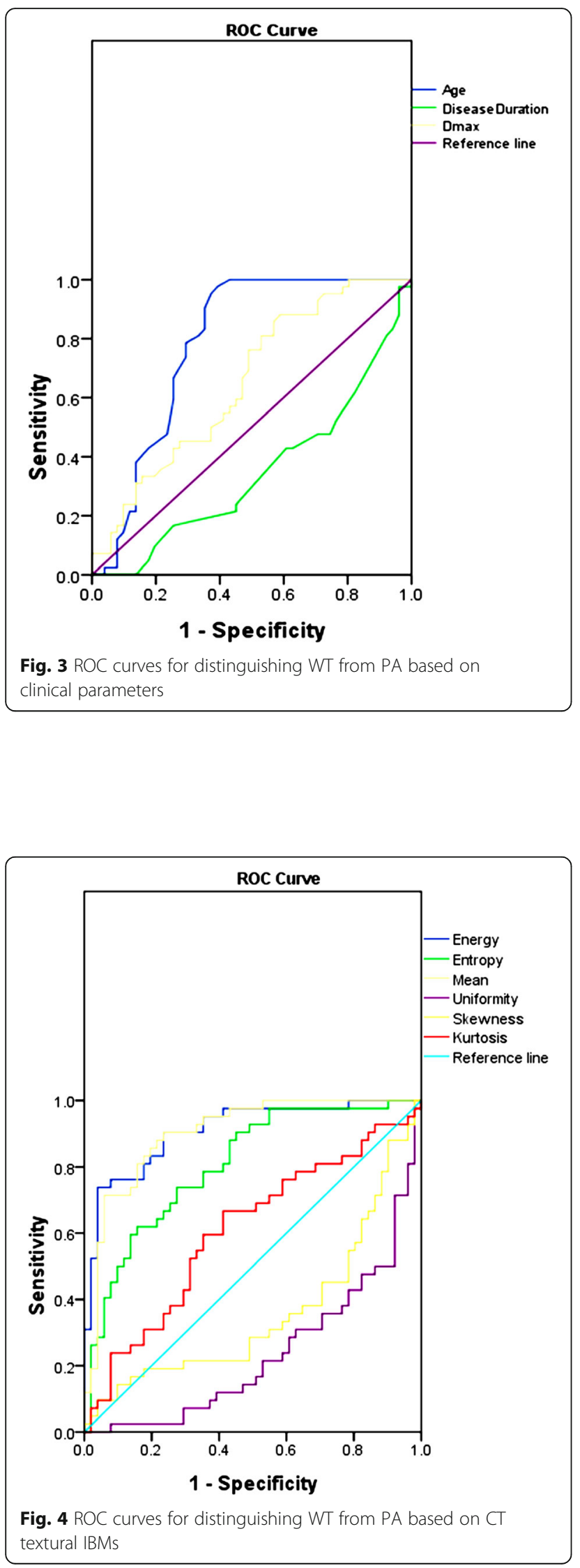

female patients from 1.7:1 to $11.5: 1$ and the average age of patients from 56.7 years to 60 years [18, 19], which was roughly consistent with the findings of the present study. While, the PA is more prone to occur in middleaged women $(P<0.001)$ compared to WT in this study, which was also in line with those found by the literature. This study found that disease duration of PA is longer than WT. We speculate that WT is larger in size $(P<$ $0.001)$ and more superficial in location $(P=0.003)$ compared with PA, which is beneficial to early detection.

Nevertheless, lots of overlapping features of WT and PA lead to the difficulties in the preoperative diagnosis [20]. Fine needle aspiration (FNA) regarded as the gold standard for the diagnosis of parotid tumors has several inevitable limitations and may be related to the low sensitivity and poor levels of diagnostic accuracy [21]. The false positive rates of FNA in PA and WT were reported to be 9 and $8 \%$, respectively, and the multifarious cytomorphology of these tumors may lead to the misdiagnosis [22]. Furthermore, the heterogeneity of tumors may be underestimated from a single or limited biopsy sample [23]. Thus, pre-operation imaging examination may be a noninvasive and better approach to identify WT and PA.

At present, multi-phase contrast-enhanced CT has been applied to the preoperative examination for the patients with parotid tumors and it has been the main method for the preoperative assessment [20, 24]. As we know, washout time of contrast agent in the tumors could provide valuable pathophysiological information and be helpful for the differential diagnosis of pariod tumors $[24,25]$. As to PA and WT, quick expurgation of contrast agent was unique for WT, while a delayed enhancement was unique for PA, which was consistent with the findings of the present study. But it was not always quite the case, for some WTs also present delayed enhancement and PAs present quick expurgation [20], which have been confirmed in this study. Furthermore, the delayed imaging would increase the radiation doses or reduce the temporal resolution.

Additionally, peripheral vessels sign was detected more frequently in WTs $(\mathrm{P}<0.001)$ compared with PAs in the arterial phase, which has not been previously reported. We speculate that WT is hypervascular lesion with abundant expanded blood capillaries according to histopathological features, which may contribute to its potential of stimulating peripheral angiogenesis.

The diagnostic efficiency of energy in all textural IBMs was the best in this cohort of parotid WT and PA patients, according to the univariate diagnostic models of this study. This was verified by the whole-volume texture analysis from CT images of arterial phase. That is to say, the textural IBM provided a stronger association with the diagnosis of WT and PA compared to clinical 
Table 4 Diagnostic performance of various indexes, including clinical parameters, conventional image features and textural IBMs

\begin{tabular}{|c|c|c|c|c|c|c|}
\hline Index & AUC & $P$ Value & $95 \% \mathrm{Cl}$ & Cutoff value & Sensitivity & Specificity \\
\hline Gender & 0.171 & $<0.01$ & $0.084-0.258$ & 1.500 & 0.048 & 0.294 \\
\hline Age & 0.784 & $<0.01$ & $0.686-0.881$ & 47.500 & 0.976 & 0.608 \\
\hline Disease duration & 0.347 & 0.011 & $0.235-0.458$ & 3.500 & 0.476 & 0.255 \\
\hline Smoking status & 0.177 & $<0.01$ & $0.088-0.267$ & 1.500 & 0.119 & 0.235 \\
\hline Site (in the tail) & 0.205 & $<0.01$ & $0.109-0.301$ & 1.500 & 0.214 & 0.196 \\
\hline Maximum diameter & 0.652 & 0.012 & $0.541-0.762$ & 1.83 & 0.881 & 0.412 \\
\hline TDC (washout type) & 0.079 & $<0.01$ & $0.014-0.145$ & 1.500 & 0.119 & 0.039 \\
\hline Peripheral vessels sign & 0.210 & $<0.01$ & $0.111-0.309$ & 1.500 & 0.381 & 0.039 \\
\hline Mean & 0.902 & $<0.01$ & $0.840-0.964$ & 67.364 & 0.905 & 0.765 \\
\hline Energy & 0.910 & $<0.01$ & $0.851-0.970$ & $42,558,500$ & 0.738 & 0.961 \\
\hline Entropy & 0.805 & $<0.01$ & $0.717-0.893$ & 1.767 & 0.738 & 0.725 \\
\hline Uniformity & 0.235 & $<0.01$ & $0.138-0.331$ & 0.211 & 1 & 0.020 \\
\hline
\end{tabular}

Abbreviations: IBMs Image biomarkers, TDC Time-density curve, AUC Area under ROC curve

model and conventional image model. The textural IBMs were quantified by extracting features from the complete tumor volume in this study, which was quite different from other texture analysis of parotid disease, for the ROI was manually drawn around the tumor on its largest cross-sectional area, instead of the whole volume of the tumor [26]. In consequence, the overall tumor features were reflected by textural IBMs. In addition to some features on plain $\mathrm{CT}$, contrast-enhanced $\mathrm{CT}$ can also reflect some heterogeneous features on tumor blood supply.

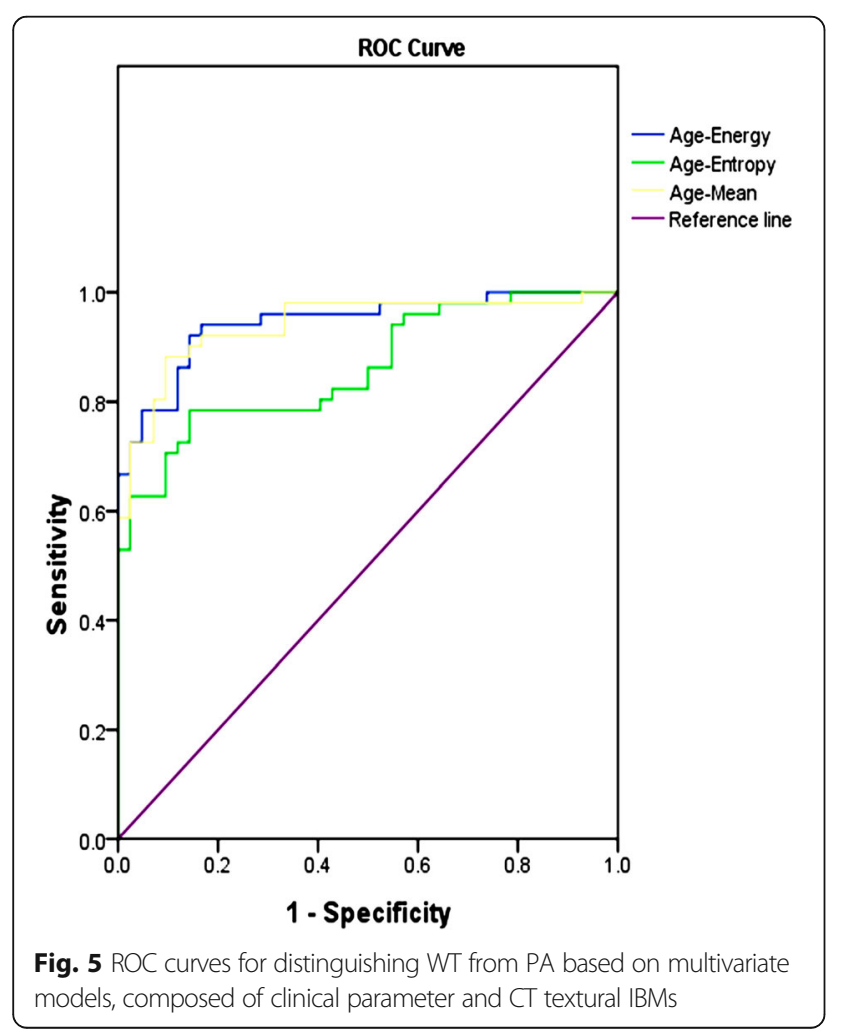

Parotid gland tumors are primarily supplied by arteries, so CT images of arterial phase were selected to analyze the texture features of PA and WT in this study.

Texture features may be acquired from several kinds of image examinations, such as $\mathrm{CT}$, magnetic resonance imaging (MRI), positron emission tomography (PET) and the like, without changing the acquisition protocols and additional costs for patients [27-29]. Currently, texture analysis is mainly used to evaluate the treatment effect and prognosis of lung cancer, colorectal cancer, liver cancer and so on [30-32]. But it is rarely applied to parotid gland, except for several reports focusing on the alterations of parotid morphology and secretion function induced by radiotherapy for head and neck cancers [33, 34]. However, as we know, there is a paucity of literature pertaining to the potential diagnostic value of CT textural IBMs in parotid tumors, as well as the multivariate model.

Given the different surgical management of PA and WT patients, it is hoped that gathering clinical clues and CT IMBs together could improve the ability to make treatment decisions and help predict surgical outcomes. In this study, the diagnostic performance was obviously improved by the combination of clinical parameter and textural IBMs, compared to univariate model, especially the conventional image model and clinical model. Our results suggest that with no additional imaging burden, texture analysis of preoperative routine contrast-

Table 5 Diagnostic performance of multivariate models, consisted of clinical parameter and textural IBMs

\begin{tabular}{llllll}
\hline Index & AUC & $P$ Value & $95 \% \mathrm{Cl}$ & Sensitivity & Specificity \\
\hline Age-Mean & 0.940 & $<0.01$ & $0.891-0.988$ & 0.882 & 0.905 \\
Age-Energy & 0.944 & $<0.01$ & $0.900-0.988$ & 0.922 & 0.857 \\
Age-Entropy & 0.861 & $<0.01$ & $0.788-0.934$ & 0.784 & 0.857 \\
\hline
\end{tabular}

Abbreviations: IBMs, Image biomarkers, AUC Area under ROC curve 
enhanced CT imaging may give us helpful information for PA and WT patients performing different operations. As we learn more about the CT textural IBM, hopefully it will lead to more benefits for new operative scheme for patients with parotid gland tumors.

The present study had several limitations. First, the sample size was relatively small, although it was larger than previous CT texture analysis studies on PA and WT [26]. Although the sample size is relatively small, we got a strong correlation between textural IBMs and the diagnosis of PA and WT, as well as the multivariate models. The results of this research inspire investigating the relationship between broader radiomic features and parotid tumors of other pathological types in a larger sample size. Second, all patients enrolled in this study were from one institution, so a large-scale randomized controlled trial needs to be performed to validate our results. At last, there is a shortage of understanding on the potential relationship between textural IBMs and histopathology of parotid PA and WT which requires further work. Meanwhile, further research needs to be done on the repeatability of these quantifiable IBMs as part of a biomarker validation process.

\section{Conclusions}

In conclusion, the multivariate models consisted of the clinical parameter and textural IBMs improved the preoperative diagnosis of WT and PA, and facilitated the individualized operation plan for patients. Consequently, the parotidectomy trauma may be minimized and the postoperative complication may be reduced. The CT textural IBMs are worth exploring to determine whether they can improve the clinical programme currently applied.

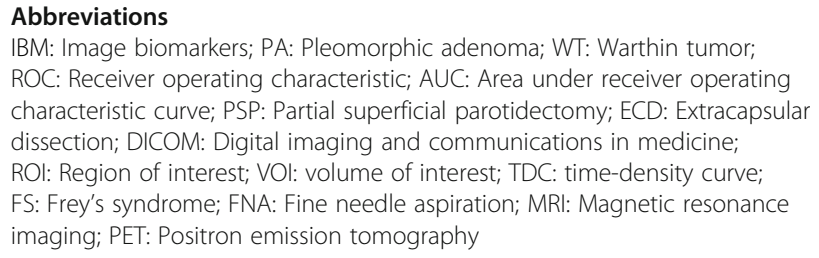

\section{Acknowledgements}

Not applicable.

\section{Authors' contributions}

ZT contributed to the study design. DZ carried out the collection and assembly of data and drafted the manuscript. DZ and HX did the feature extraction and LL, JY did statistical work. XL1, RL and CY prepared Figs. XH, BZ and XL2 participated in the design of the study and helped to draft the manuscript. All authors reviewed the manuscript. All authors read and approved the final manuscript.

\section{Funding}

This work was supported by several organizations in the data analysis and manuscript revision, including the medical research Key Program of the combination of Chongqing National health commission and Chongqing science and technology bureau, China (No.2019ZDXM010), the Basic and Frontier Research Project of Chongqing, China (No.cstc2016jcyjA0294), the
Medical Research Key Program of the National Health and Family Planning Commission of Chongqing, China.

(No.20141016 and No.2016ZDXM026), the Scientific and Technological Innovation Key Program of Chongqing General Hospital, China

(No.2016ZDXM03).

\section{Availability of data and materials}

The datasets used and analysed during the current study are available from the corresponding author on reasonable request.

\section{Ethics approval and consent to participate}

This study was approved by the local Ethics Committee of the First Affiliated Hospital of Chongqing Medical University, and formal consent is not required, for it is a retrospective study.

\section{Consent for publication}

The manuscript is approved by all participants for publication.

\section{Competing interests}

The authors declare that they have no competing interests.

\section{Author details}

'Department of Radiology, Chongqing General Hospital, University of Chinese Academy of Sciences, No.104 Pipashan Main St, Yuzhong District, Chongqing 400014, China. ${ }^{2}$ Molecular and Functional Imaging Laboratory, Chongqing General Hospital, University of Chinese Academy of Sciences, Chongqing 400014, China. ${ }^{3}$ Department of Radiology, The First Affiliated Hospital of Chongqing Medical University, Chongqing 400016, China.

Received: 16 December 2019 Accepted: 7 April 2020

Published online: 15 April 2020

\section{References}

1. Izzo L, Casullo A, Caputo M, et al. Space occupying lesions of parotid gland. Comparative diagnostic imaging and pathological analysis of echo color /power Doppler and of magnetic resonance imaging. Acta Otorhinolaryngol Ital. 2006;26(3):147-53.

2. Hilton JM, Phillips JS, Hellquist HB, et al. Multifocal multi-site Warthin tumour. Eur Arch Otorhinolaryngol. 2008;265(12):1573-5. https://doi.org/10. 1007/s00405-008-0622-z.

3. Chulam TC, Noronha Francisco AL, Goncalves Filho J, et al. Warthin's tumour of the parotid gland: our experience. Acta Otorhinolaryngol Ital. 2013;33(6):393-7.

4. Yu C, Song Z, Xiao Z, et al. Mucoepidermoid carcinoma arising in Warthin's tumor of the parotid gland: clinicopathological characteristics and immunophenotypes. Sci Rep. 2016;6:30149. https://doi.org/10.1038/ srep30149.

5. Iro H, Zenk J. Role of extracapsular dissection in surgical management of benign parotid tumors. JAMA Otolaryngol Head Neck Surg. 2014;140(8): 768-9. https://doi.org/10.1001/jamaoto.2014.1218.

6. Park SJ, Han S, Lee HJ, et al. Preservation of salivary function following extracapsular dissection for tumors of the parotid gland. J Oral Maxillofac Surg. 2018;76(9):2004-10. https://doi.org/10.1016/j.joms.2018.03.033.

7. van Dijk LV, Brouwer $\mathrm{CL}$, van der Schaaf $\mathrm{A}$, et al. CT image biomarkers to improve patient-specifc prediction of radiation-induced xerostomia and sticky saliva. Radiother Oncol. 2016;122(2):185-91. https://doi.org/10.1016/j. radonc.2016.07.007.

8. Nardone $V$, Tini $P$, Nioche $C$, et al. Texture analysis of parotid gland as a predictive factor of radiation induced xerostomia: a subset analysis. Radiother Oncol. 2017;122(2):321. https://doi.org/10.1016/j. radonc.2016.09.004

9. Cui Y, Song J, Pollom E, et al. Quantitative analysis of (18) Ffluorodeoxyglucose positron emission tomography identifies novel prognostic imaging biomarkers in locally advanced pancreatic cancer patients treated with stereotactic body radiation therapy. Int J Radiat Oncol Biol Phys. 2016;96(1):102-9. https://doi.org/10.1016/j.jirobp. 2016.04.034.

10. Chen S, Zhu Y, Liu Z, et al. Texture analysis of baseline multiphasic hepatic computed tomography images for the prognosis of single hepatocellular carcinoma after hepatectomy: a retrospective pilot study. Eur J Radiol. 2017; 90:198-204. https://doi.org/10.1016/j.ejrad.2017.02.035. 
11. Canellas R, Burk KS, Parakh A, et al. Prediction of pancreatic neuroendocrine tumor grade based on CT features and texture analysis. AJR Am J Roentgenol. 2018;210(2):341-6. https://doi.org/10.2214/AJR.17.18417.

12. Liu J, Mao Y, Li Z, et al. Use of texture analysis based on contrast-enhanced MRI to predict treatment response to chemoradiotherapy in nasopharyngeal carcinoma. J Magn Reson Imaging. 2016;44(2):445-55. https://doi.org/10.1002/jmri.25156.

13. Ciuman RR, Oels $W$, Jaussi R, et al. Outcome, general, and symptom-specific quality of life after various types of parotid resection. Laryngoscope. 2012; 122(6):1254-61. https://doi.org/10.1002/lary.23318.

14. Tweedie DJ, Jacob A. Surgery of the parotid gland: evolution of techniques, nomenclature and a revised classification system. Clin Otolaryngol. 2009; 34(4):303-8. https://doi.org/10.1111/j.1749-4486.2009.01953.x.

15. Kato MG, Erkul E, Nguyen SA, et al. Extracapsular dissection vs superficial parotidectomy of benign parotid lesions surgical outcomes and costeffectiveness analysis. JAMA Otolaryngol Head Neck Surg. 2017;143(11): 1092-7. https://doi.org/10.1001/jamaoto.2017.1618.

16. Hamilton BE, Salzman $\mathrm{KL}$, Wiggins $\mathrm{RH}$, et al. Earring lesions of the parotid tail. AJNR Am J Neuroradiol. 2003;24(9):1757-64.

17. Witt RL, Rejto L. Pleomorphic adenoma: extracapsular dissection versus partial superficial parotidectomy with facial nerve dissection. Del Med J. 2009;81(3):119-25.

18. Patel DK, Morton RP. Demographics of benign parotid tumours: Warthin's tumour versus other benign salivary tumours. Acta Otolaryngol. 2016;136(1): 83-6. https://doi.org/10.3109/00016489.2015.1081276.

19. Wang CW, Chu YH, Chiu DY, et al. The Warthin tumor score: a simple and reliable method to distinguish Warthin tumors from pleomorphic adenomas and carcinomas. AJR Am J Roentgenol. 2018;210(6):1330-7. https://doi.org/10.2214/AJR.17.18492.

20. Xu ZF, Yong F, Yu T, et al. Different histological subtypes of parotid gland tumors: CT findings and diagnostic strategy. World J Radiol. 2013;5(8):31320. https://doi.org/10.4329/wjr.v5.i8.313.

21. Jo HJ, Ahn HJ, Jung S, et al. Diagnostic difficulties in fine needle aspiration of benign salivary glandular lesions. Korean J Pathol. 2012;46(6):569-75. https://doi.org/10.4132/koreanjpathol.2012.46.6.569

22. Kechagias N, Ntomouchtsis A, Valeri R, et al. Fine-needle aspiration cytology of salivary gland tumours: a 10-year retrospective analysis. Oral Maxillofac Surg. 2012;16(1):35-40. https://doi.org/10.1007/s10006-011-0291-8.

23. Lipinski KA, Barber $L$, Davies MN, et al. Cancer evolution and the limits of predictability in precision cancer medicine. Trends Cancer. 2016;2(1):49-63. https://doi.org/10.1016/j.trecan.2015.11.003.

24. Woo SH, Choi DS, Kim JP, et al. Two-phase computed tomography study of Warthin tumor of parotid gland: differentiation from other parotid gland tumors and its pathologic explanation. J Comput Assist Tomogr. 2013;37(4): 518-24. https://doi.org/10.1097/rct.0b013e31828aede8.

25. Dong $Y$, Lei GW, Wang SW, et al. Diagnostic value of $C T$ perfusion imaging for parotid neoplasms. Dentomaxillofac Radiol. 2014;43(1):20130237. https:// doi.org/10.1259/dmfr.20130237

26. Al Ajmi E, Forghani B, Reinhold C, et al. Spectral multi-energy CT texture analysis with machine learning for tissue classification: an investigation using classification of benign parotid tumours as a testing paradigm. Eur Radiol. 2018;28(6):2604-11. https://doi.org/10.1007/s00330-017-5214-0.

27. Koo HJ, Sung YS, Shim WH, et al. Quantitative computed tomography features for predicting tumor recurrence in patients with surgically resected adenocarcinoma of the lung. PLoS One. 2017;12(1):e0167955. https://doi. org/10.1371/journal.pone.0167955.

28. Zhai TT, Langendijk JA, van Dijk LV, et al. The prognostic value of CT-based image-biomarkers for head and neck cancer patients treated with definitive (chemo-)radiation. Oral Oncol. 2019;95:178-86. https://doi.org/10.1016/j. oraloncology. 2019.06.020.

29. Eilaghi A, Baig S, Zhang Y, et al. CT texture features are associated with overall survival in pancreatic ductal adenocarcinoma - a quantitative analysis. BMC Med Imaging. 2017;17(1). https://doi.org/10.1186/s12880-0170209-5.

30. Simpson AL, Doussot A, Creasy JM, et al. Computed tomography image texture: a noninvasive prognostic marker of hepatic recurrence after hepatectomy for metastatic colorectal cancer. Ann Surg Oncol. 2017;24(9): 2482-90. https://doi.org/10.1245/s10434-017-5896-1.

31. Li Z, Mao Y, Huang W, et al. Texture-based classification of different single liver lesion based on SPAIR T2W MRI images. BMC Med Imaging. 2017;17(1): 42. https://doi.org/10.1186/s12880-017-0212-X.
32. Pham TD, Watanabe $Y$, Higuchi M, et al. Texture Analysis and Synthesis of Malignant and Benign Mediastinal Lymph Nodes in Patients with Lung Cancer on Computed Tomography. Sci Rep. 2017;7:43209. https://doi.org/ 10.1038/srep43209.

33. Nardone $V$, Tini $P$, Nioche $C$, et al. Texture analysis as a predictor of radiation-induced xerostomia in head and neck patients undergoing IMRT. Radiol Med. 2018;123(6):415-23. https://doi.org/10.1007/s11547-017-0850-7.

34. Wu H, Chen $X$, Yang $X$, et al. Early prediction of acute xerostomia during radiation therapy for head and neck cancer based on texture analysis of daily CT. Int J Radiat Oncol Biol Phys. 2018;102(4):1308-18. https://doi.org/ 10.1016/j.jijobp.2018.04.059.

\section{Publisher's Note}

Springer Nature remains neutral with regard to jurisdictional claims in published maps and institutional affiliations.
Ready to submit your research? Choose BMC and benefit from:

- fast, convenient online submission

- thorough peer review by experienced researchers in your field

- rapid publication on acceptance

- support for research data, including large and complex data types

- gold Open Access which fosters wider collaboration and increased citations

- maximum visibility for your research: over $100 \mathrm{M}$ website views per year

At BMC, research is always in progress.

Learn more biomedcentral.com/submissions 